\title{
Conventional Scalpel vs Laser Biopsy: A Comparative Pilot Study
}

\author{
${ }^{1}$ MS Munisekhar, ${ }^{2} \mathrm{~K}$ Mahendranadh Reddy, ${ }^{3}$ Syed Afroz Ahmed, ${ }^{4}$ Charu Suri, ${ }^{5}$ Esther Priyadarshini S \\ ${ }^{1}$ Professor and Head, Department of Oral and Maxillofacial Pathology, Sri Sai College of Dental Sciences, Vikarabad, Andhra Pradesh, India \\ ${ }^{2}$ Principal, Sri Sai College of Dental Sciences, Vikarabad, Andhra Pradesh, India \\ ${ }^{3}$ Professor, Department of Oral and Maxillofacial Pathology, Sri Sai College of Dental Sciences, Vikarabad, Andhra Pradesh, India \\ ${ }^{4}$ Reader, Department of Oral and Maxillofacial Pathology, Sri Sai College of Dental Sciences, Vikarabad, Andhra Pradesh, India \\ ${ }^{5}$ Postgraduate Student, Department of Oral and Maxillofacial Pathology, Sri Sai College of Dental Sciences, Vikarabad, Andhra Pradesh, India
}

Correspondence: K Mahendranath Reddy, Principal, Sri Sai College of Dental Sciences, Vikarabad, Andhra Pradesh, India e-mail: mrkareti@gmail.com

\section{ABSTRACT}

During a biopsy procedure, it is fundamental to maintain safe and readable cut margins in order to permit histological visualization of a lesion. With the advent of lasers, laser surgery has been shown to exhibit several advantages over scalpel surgery. But, there has been a considerable controversy concerning the reliability of laser biopsies and the effects of thermal damage to the edge of the laser wounds. The aim of this pilot study was to compare and evaluate the efficacy of laser biopsy to conventional method with regard to histological peripheral damage. In this study, Waterlase (Er, Cr: YSGG, $2780 \mathrm{~nm})$ and Diode lasers $(940 \mathrm{~nm})$ at different power settings and fluences were used. In the biopsy specimens, the cut edges of the incision, when lower settings were applied, were without any adverse effects and there was no difficulty in diagnosis. But whereas in the specimens obtained using higher settings diagnosis was difficult.

Keywords: Diagnosis, Hemostasis, Histology.

\section{INTRODUCTION}

A biopsy is a surgical procedure performed to establish a clear diagnosis of a lesion and for the subsequent planning of an appropriate treatment. A biopsy may be either incisional or excisional. An incisional biopsy is done to remove one or more pathological samples so that adequate therapy can be started after histopathological evaluation. By contrast, an excisional biopsy involves complete removal of the lesion, so it is a diagnostic and therapeutic procedure. During a biopsy procedure, it is fundamental that the cut margins are readable so that possible marginal infiltrations or any benign to malignant transformation of a lesion can be histologically evaluated.

Traditionally, conventional scalpel biopsies have been used to establish:

1. Histological characteristics of suspect lesions

2. Their differentiation

3. Extent or spread and

4. To adopt an adequate treatment strategy. ${ }^{1}$

After the advent of lasers, the promotion, popularity and successful outcome of laser treatment in areas of medical health care have made patients more inquisitive about the use of lasers in dentistry. ${ }^{2}$ Lasers are employed for numerous purposes in various fields of science and the most advantageous use is found in the medical and dental arena.

Though lasers were first introduced in dentistry in 1964, they were not widely used in oral applications until around 1980. As quoted by Davide Zaffe et al, initially studies were carried out on ruby laser applicability in dentistry by Goldman et al, ${ }^{3}$ Taylor ${ }^{4}$ and Stern et al, ${ }^{5}$ followed by several studies which were carried out on the clinical use of different lasers, like argon, $\mathrm{CO}_{2}$, neodymium-doped: Yttrium, aluminium, garnet and erbium-doped: Yttrium, aluminium and garnet lasers. ${ }^{6}$

Laser is defined as "a device which amplifies electromagnetic energy at various optical frequencies into an extremely intense, small and nearly nondivergent beam of bright light of a single color”. Laser is capable of mobilizing intense heat and power when focused at a close range and it is used as a tool in surgical procedures, in diagnosis and physiological studies.

The word Laser is an acronym for light amplification by stimulated emission of radiation. ${ }^{7}$

\section{APPLICATIONS IN DENTISTRY}

Traditionally, lasers have been widely used in dentistry for cavity and root canal preparations, coagulation and hemostasis, exposure of implants, scaling and root planing, gingival and periodontal surgeries, biopsies, excision of tongue lesions, TMJ disorders and preprosthetic surgery.

But due to the considerable controversy concerning the reliability of laser biopsies and the effects of thermal damage to the edge of laser wounds, we evaluated the effects of the use of different lasers (diode $940 \mathrm{~nm}$ ) and Er, Cr:YSGG (2780 $\mathrm{nm}$ ) on histological diagnosis in vivo. 


\section{AIMS AND OBJECTIVES}

Numerous studies have been carried out on using lasers for obtaining biopsies and to evaluate their effects on oral tissues. However, the results obtained were variable and inconclusive. Hence, this pilot study was carried out to evaluate the effect of lasers specifically (diode laser, $940 \mathrm{~nm}$ and Er, Cr:YSGG, 2780 $\mathrm{nm}$ ) on the peripheral architecture of the suspected oral lesions.

\section{MATERIALS AND METHODS}

The study was performed on six patients after obtaining clearance from the Institutional Ethical Committee to evaluate the peripheral effects induced by diode $(940 \mathrm{~nm})$ and Er, Cr:YSGG $(2780 \mathrm{~nm})$ lasers on oral soft tissues. Necessary precautions and safety protocols were undertaken by the operator and the patient all through the operating procedure.

1. Two biopsy specimens of 1sq.cm were obtained from two cases of suspected oral squamous cell carcinoma by using Er, Cr: YSGG (Waterlase) with the power settings (power $2 \mathrm{~W}$, wavelength $2780 \mathrm{~nm}, 30 \mathrm{pps}$ )

2. Two biopsy specimens of 1 sq.cm were obtained from two cases of suspected oral squamous cell carcinoma by using high power diode laser (ezLase) with the power settings (power 2W, wavelength $940 \mathrm{~nm}, 30 \mathrm{pps}$ )

3. A biopsy specimen of 1 sq.cm was obtained from a suspected case of oral submucous fibrosis (OSMF) by using high power diode laser with the power settings (power $3 \mathrm{~W}$, wavelength $940 \mathrm{~nm}, 30 \mathrm{pps}$ )

\section{RESULTS (FIGS 1A TO 4B)}
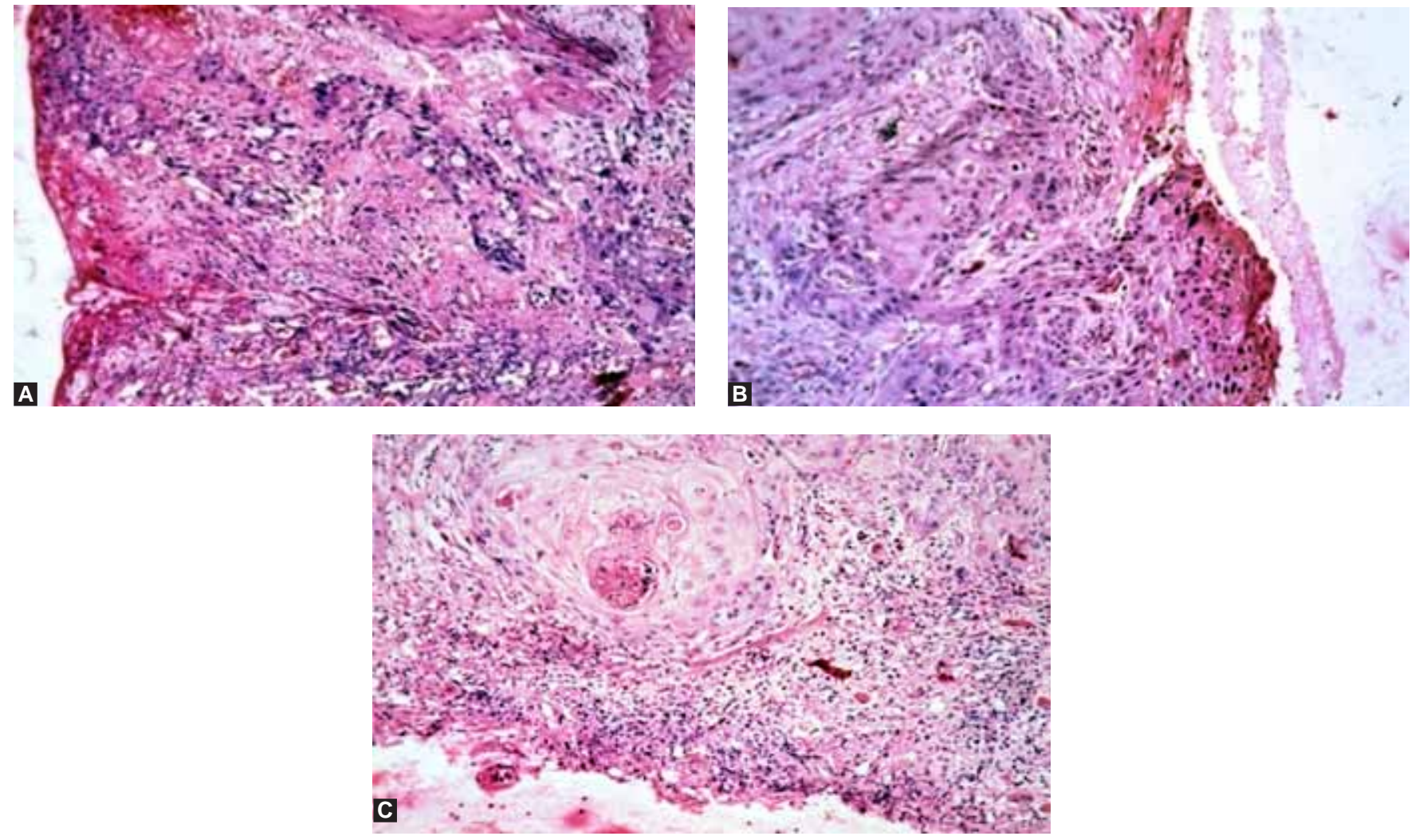

Figs 1A to C: Result using waterlase: (A) Case $1(4 \times)$ (B) Case $1(10 x)$ (C) Case $2(10 x)$
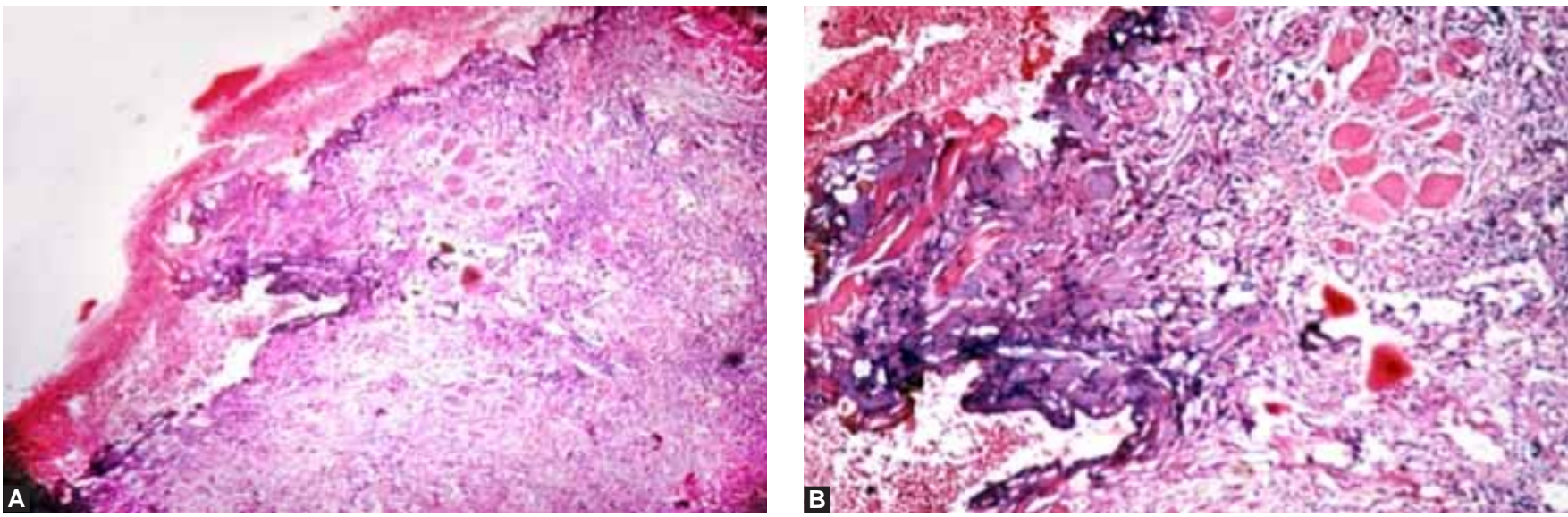

Figs 2A and B: Results using low frequency diode laser: (A) Case $1(4 \times)$ (B) Case $1(10 x)$ 

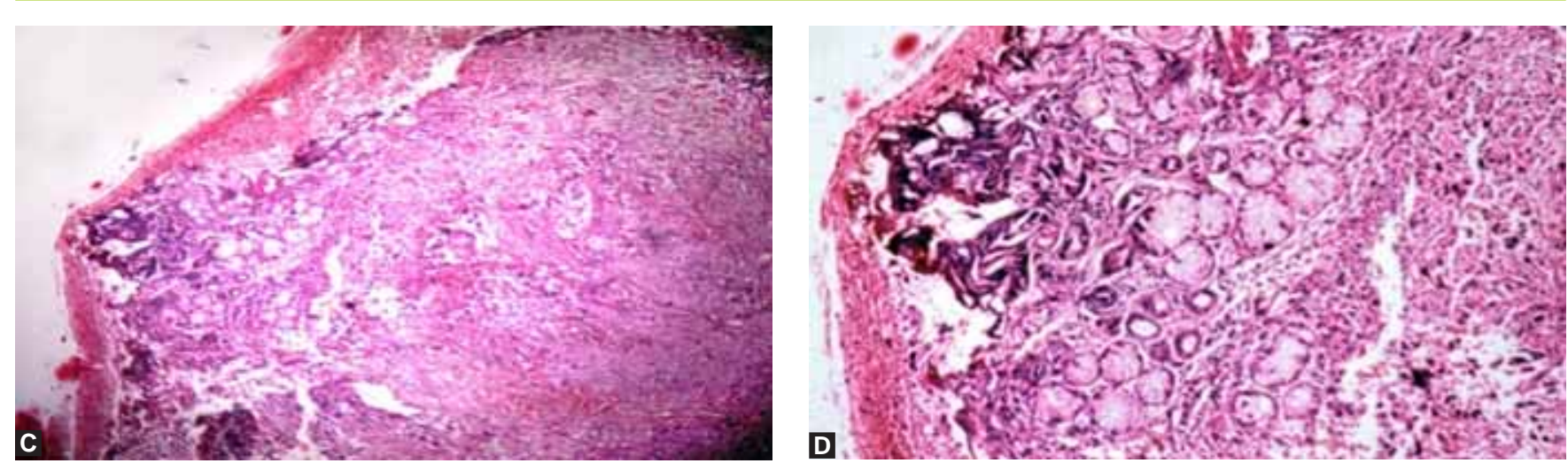

Figs 2C and D: Results using low frequency diode laser: (C) Case 2 (4x) (D) Case 2 (10x)
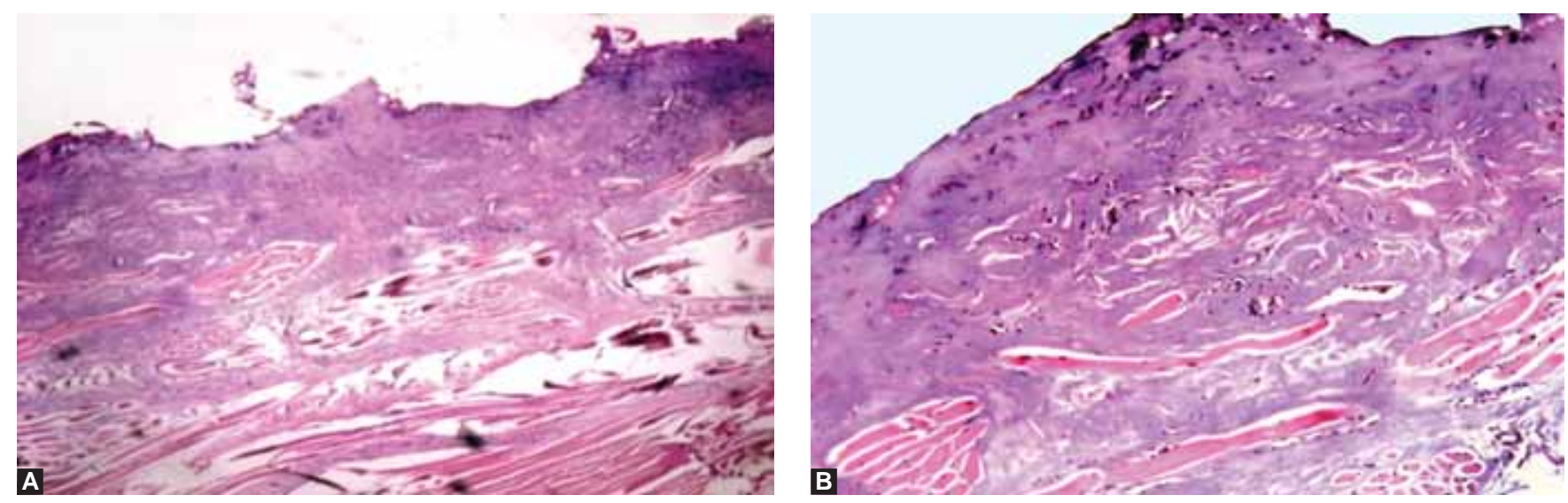

Figs 3 A and B: Results using high frequency diode laser: (A) $4 \times$ (B) $10 \times$
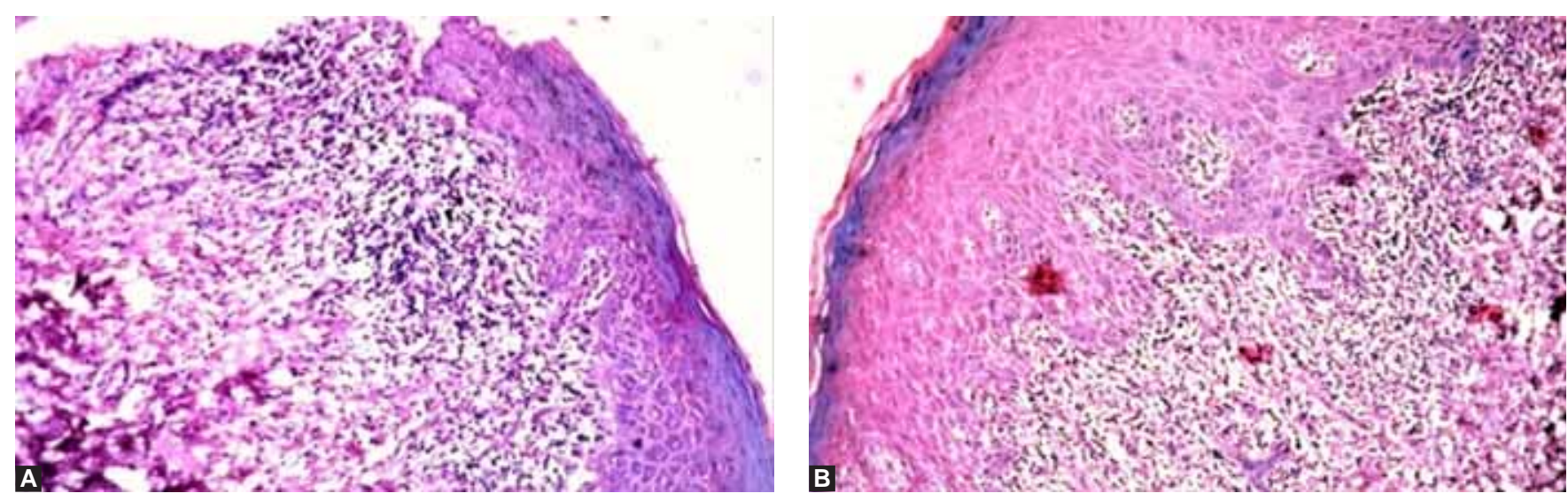

Figs 4A and B: Results using conventional biopsy (Control): (A) $4 \times$ (B) 10×

4. A biopsy specimen of 1sq.cm was taken using a Bard-Parker no.15 blade to serve as a control.

Following the excision, the specimens were placed in $10 \%$ formalin solution for adequate fixation. The specimens were embedded in paraffin and $3 \mu \mathrm{m}$ sections were obtained. The hematoxylin and eosin stained sections were then examined separately by two different evaluators to rule out any interobserver bias.

\section{DISCUSSION}

Biopsies are recommended whenever a clinician encounters a suspicious lesion without a definitive diagnosis. Biopsies are traditionally being performed using conventional scalpel and blade. However, with the introduction of lasers to dentistry, laser biopsies too are being performed with promising results. Though lasers are primarily used for excision of lesions (excisional biopsy), it can also be used for diagnostic purposes. Different kinds of lasers are being used in dentistry, like ruby, argon laser, diode laser, $\mathrm{CO}_{2}$ laser, $\mathrm{Nd}$ :YAG lasers, etc. Literature revealed that of all different lasers available commercially $\mathrm{CO}_{2}$ lasers seem to be laser of choice by surgeons. ${ }^{8}$

\section{Principle}

All surgical dental lasers function through a wavelengthspecific photonic absorption which increases the temperature within the target tissues. ${ }^{9}$ Laser beam increases the temperature 
at a point of incidence by more than $100^{\circ} \mathrm{C}$. Hence, lasers cut by heating the tissues. Therefore, one of the undesired effects that lasers induce is irreversible or permanent damage due to thermal reaction in the surrounding tissues. ${ }^{10}$

On the other hand, lasers have added advantages, like less bleeding (hemostasis), less pain, reduced risk of infection, sealsoff nerve endings and lymphatic vessels and minimize the need for suturing.

Our study was carried out by using Er., Cr: YSGG (2780 $\mathrm{nm})$ (Waterlase) and diode (940 nm) (ezlase) laser to find out thermal changes introduced in the tissue of interest and to know whether histological diagnosis was possible from such tissues. Though literature reveals $\mathrm{CO}_{2}$ lasers are the best lasers for oral soft tissues, ${ }^{8}$ the above-mentioned lasers were utilized for our study as they were available and routinely used in our institution. An attempt was made to analyze the thermal effects particularly at the periphery/edge.

In the tissue sections obtained from biopsy specimens cut by Waterlase ( $2780 \mathrm{~nm}$ ) laser, the margins of the tissue sections, were practically without any adverse effects both in the epithelium as well as in the underlying connective tissue in both the cases. They were comparable to tissue sections obtained by scalpel biopsy and there was no difficulty in diagnosing the suspected lesions. This is probably because the Er, Cr:YSGG (Waterlase) (2780 nm) laser beam induces minimal thermal effects and therefore does not cause any coagulation of the tissues in areas of our interest. Hence, when biopsies were taken with these lasers, bleeding from the cut tissues was observed which was comparable to a scalpel biopsy.

Literature also showed that diode lasers when used cause sufficient tissue damage, like tissue necrosis and/or sloughing and charring of the tissue margins. ${ }^{9}$ But, in contrary to this, the tissue sections obtained from biopsy specimens cut by diode laser using relatively low power settings (P-2.0W, $\lambda-940 \mathrm{~nm}$, $30 \mathrm{pps}$ ), the margins of the lesion and also the epithelium and the underlying connective tissue as a whole did not show any undesired effects caused by the use of lasers. However, in focal areas at the margins associated with epithelium, areas of coagulation were noticed. Yet, in both the cases, the diagnosis of the lesion from such sections by both the evaluators was not difficult. Hence, tissues obtained by diode lasers using low power settings though contained few undesirable changes, could still be diagnosed. But such minor thermal damage at the margins may pose problems in determining the extent of the lesion as well as diagnosis of dysplastic/neoplastic lesions. Hence, it is advisable to make the incision well beyond the margins of the suspected lesion so that the evaluator is totally free from uncertainty and does not misinterpret the histological picture.

But when diode laser was used with higher power settings to obtain a biopsy specimen from a suspected OSMF case as it was difficult to cut with low power settings, clinically, there was complete charring of the tissues at the periphery. In addition, histologically, the margins of the cut sections showed complete coagulation and were without overlying epithelium. In this case, no diagnosis was possible. Though the exact reason for the loss of epithelium is not known, it is probable that the heat generated by the diode laser with high power settings could have led to separation of epithelium from the underlying connective tissue and subsequently lost during tissue processing. However, more tissue samples with this setting have to be carried out for a definitive assessment.

From our study, we are in agreement with Mitchell AL that a clinician always should use the lowest possible power settings to get the intended objective and that by increasing power settings, only to cut tissue faster may lead to adverse effects making tissue specimen not acceptable for diagnostic purpose defeating the advantage of using dental laser.

\section{CONCLUSION}

The study is an attempt to throw more light on the use of lasers for oral biopsy and its effectiveness. Our study revealed that Er, Cr:YSGG (2780 nm) (Waterlase) has been more effective by producing least tissue distortion/artefacts. However, to minimize the artefacts associated with any laser, we stress that the operator should observe adequate care so that results are as close as possible to a scalpel biopsy. To come to a more definitive conclusion, a study with a large sample size is being carried out in continuation of this pilot study.

\section{REFERENCES}

1. AmparoMota-Ramirez, Francisco Javier Silvestre, Juan Manuel Simo. Oral biopsy in dental practice. Med Oral Patol Cir Bucal 2007;12(7):504-10.

2. Scott D Benjamin. Focus on lasers soft tissue lasers: It's the wavelength, power, ergonomics, and control that matter 2009.

3. Goldman L, Hornby P, Meyer R. Impact of the laser on dental caries. Nature 1964;203:417.

4. Richard Taylor, Gerald Shklar, Fred Roeber BS. The effects of laser radiation on teeth, dental pulp, and oral mucosa of experimental animals. Oral Surgery, Oral Medicine, Oral Pathology. June 1965;19(6):786-95.

5. Stern RH, Sognnaes RF. Laser beam effect on dental hard tissue. J Dent Res 1964;43:873.

6. DavideZaffe, Marina C, Vitale. Morphological, histochemical, and immunocytochemical study of $\mathrm{CO}_{2}$ and Er:YAG laser effect on oral soft tissues. Photomedicine and laser surgery. June 2004;22(3):185-89.

7. Leo J Miserendino, Robert M Pick. Lasers in dentistry. Quintessence Publishing Co, Inc 1995.

8. Robert A Strauss, Steven D Fallon. Lasers in contemporary oral and maxillofacial surgery. Dent Clin N Am 2004;48:861-88.

9. Mitchell A. Lomke. Clinical applications of dental lasers. General Dentistry 2009:47-59.

10. Umberto Romeo, GasperePalaia, Alessandro Del Vecchio. Effects of KTP laser on oral soft tissues. An in vitro study. Lasers Med Sci 2010. 